\title{
Infrared spectroscopy of a brown dwarf companion candidate near the young star GSC 08047-00232 in Horologium ${ }^{\star}$
}

\author{
R. Neuhäuser ${ }^{1}$ and E. W. Guenther ${ }^{2}$ \\ 1 Astrophysikalisches Institut, Universität Jena, Schillergäßchen 2-3, 07745 Jena, Germany \\ 2 Thüringer Landessternwarte Tautenburg, Sternwarte 5, 07778 Tautenburg, Germany
}

Received 19 November 2003 / Accepted 3 March 2004

\begin{abstract}
We present infrared $H$ - and $K$-band spectra of a companion candidate 3 " north of the young star GSC 08047-00232, a probable member of the nearby young Horologium association. From previously obtained $J H K$-band colors and the magnitude difference between primary and companion candidate, the latter could well be substellar (Neuhäuser et al. 2000) with the spectral type being roughly M 7-L 9 from the $J H K$ colors (Chauvin et al. 2003). With the $H$-and $K$-band spectra now obtained with ISAAC at the VLT, the spectral type of the companion candidate is found to be M 6-9.5. Assuming the same age and distance as for the primary star ( $\sim 35 \mathrm{Myr}, 50$ to $85 \mathrm{pc}$ ), this yields a mass of $\sim 25$ Jupiter masses for the companion, hence indeed substellar. After TWA-5 B and HR 7329 B, this is the third brown dwarf companion around a nearby $(\leq 100 \mathrm{pc})$ young $(\leq 100 \mathrm{Myr}$ ) star. A total of three confirmed brown dwarf companions (any mass, separation $\geq 50 \mathrm{AU}$ ) around 79 stars surveyed in three young nearby associations corresponds to a frequency of $6 \pm 4 \%$ (with a correction for missing companions which are almost on the same line-of-sight as the primary star instead of being separated well), consistent with the expectation, if binaries have the same mass function as field stars. Hence, it seems that there is no brown dwarf desert at wide separations.
\end{abstract}

Key words. stars: late-type - stars: low-mass, brown dwarfs - stars: pre-main sequence

\section{Introduction: Young brown dwarf companions}

Brown dwarfs (BDs) are objects with mass below the hydrogen burning mass limit, i.e. below $\sim 0.078 M_{\odot}$ (e.g., Burrows et al. 1997). It is not yet clear, how BDs form. According to one of the suggested scenarios, accreting companions get ejected as BD embryos by encounters inside a forming multiple system (e.g., Reipurth \& Clarke 2001). In such a case, BDs may be different from stars regarding multiplicity, kinematics, and disk properties, so that such a scenario can be tested observationaly, e.g. by comparing the secular evolution of star-star binaries with star-BD binaries: If single BDs became single by the ejection from a multiple system, then the fraction of star-BD binaries should decrease faster with time than star-star binaries.

A possible secular evolution in stellar binaries has been studied by Bouvier et al. (2001) and Patience et al. (2002) with as yet inconclusive results. In order to compare such results with star-BD binary fractions, one would first need significant statistics. Hence, several groups search for BDs as companions to stars.

As far as young stars ( $\leq 100 \mathrm{Myr})$ in nearby associations $(\leq 100 \mathrm{pc})$ are concerned, only two brown dwarfs have been

\footnotetext{
Send offprint requests to: R. Neuhäuser, e-mail: rne@astro.uni-jena.de

* Based on observations obtained on Cerro Paranal, Chile, in ESO programs 65.L-0144 and 71.C-0206C.
}

confirmed so far as companions by both common proper motion as well as spectroscopy, namely TWA-5 B (Lowrance et al. 1999; Neuhäuser et al. 2000) in the TW Hya association (TWA, Webb et al. 1999) and HR 7329 B (Lowrance et al. 2000; Guenther et al. 2001) in the Horologium association (HorA, Torres et al. 2000) and/or $\beta$ Pic moving group (Zuckerman \& Webb 2000).

In Sect. 2, we present previous and new imaging observation and investigate the astrometry, i.e. whether the pair is co-moving. Our spectroscopic observations, the data reduction, and the final $H$ - and $K$-band spectra are shown in Sect. 3. We obtain a mass estimate for the companion and interprete the results in Sect. 4. A discussion in terms of BD companion frequency is given in the last section.

\section{Imaging observation: A companion candidate}

Recently, both Neuhäuser et al. (2000, henceforth N03) and Chauvin et al. (2003, henceforth C03) have searched for faint companions to confirmed and probable members of the young Horologium and Tucana associations, which may form one common association (TucHorA). N03 used the normal infrared (IR) imaging camera SOFI and the MPE speckle camera Sharp, both at the ESO-3.5 m-NTT, while C03 used the ADONIS AO system at the ESO-3.6 m telescope. Their samples are partly overlaping and partly disjunct, e.g. N03 also 
Table 1. Astrometry and photometry.

\begin{tabular}{llcccccc}
\hline \hline Date & Instrument & Sep. ['] & PA [deg] & $J[\mathrm{mag}]$ & $H[\mathrm{mag}]$ & $K[\mathrm{mag}]$ & Ref. \\
\hline 04 July 2001 & NTT-Sharp & $3.238 \pm 0.022$ & $357.65 \pm 0.18$ & & & $15.0 \pm 0.3$ & N03 \\
29 Oct. 2001 & 3.6 m-Adonis & $3.210 \pm 0.118$ & $359.2 \pm 2.3$ & $16.25 \pm 0.25$ & $15.2 \pm 0.18$ & $14.9 \pm 0.2$ & C03 \\
20 July 2003 & VLT-ISAAC & $3.138 \pm 0.048$ & $\sim 358$ & (narrow band filter) & & here \\
\hline
\end{tabular}

included $\beta$ Pic members. Both groups detected a promising companion candidate $3^{\prime \prime}$ north of GSC 08047-00232, a K3 V star suggested to be a Horologium member by Torres et al. (2000) with $\mathrm{H} \alpha$ emission and a lithium equivalent width of $0.35 \AA$, located at $\alpha=01^{\mathrm{h}} 52^{\mathrm{m}} 14.63^{\mathrm{s}}$ and $\delta=-52^{\circ} 19^{\prime} 33.1^{\prime \prime}(\mathrm{J} 2000.0)$; the star is also called TYC 8047-232-1 (Tycho, Hog et al. 2000), ERX 14 (Torres et al. 2000), and 1RXSJ015215.7-521939 (ROSAT, Voges et al. 1999). The position in the sky, the radial velocity, the proper motion, and the kinematical parallaxe (and its position in the $\mathrm{H}-$ $\mathrm{R}$ diagram at that distance) are all consistent with membership to HorA (Torres et al. 2000) and also with the possible common TucHor association. Also, the youth indicators like $\mathrm{H} \alpha$ emission, lithium absorption, X-ray emission, and rotational velocity are also consistent qualitatively and quantitatively with an age of roughly 35 Myr as HorA/TucHorA (Torres et al. 2000).

Given the $J H K$-band colors, C03 found that the companion candidate could have a spectral type between M 7 and L 9 by comparing the Leggett et al. (2002) photometry with the Geballe et al. (2002) spectral classifications. From the magnitude difference in $K$, also N03 concluded that the companion candidate could be a BD companion of late-M or $\mathrm{L}$ spectral type.

To confirm or reject its companion status, one can either try to (i) take a spectrum, or (ii) prove the pair to be co-moving, or (iii) show the radial velocity or astrometric wobble of the primary due to the companion (both very small), or (iv) detect orbital motion (after a long time). Only the first option is immediately possible to achieve, given the fact that the proper motion of the primary star is very small $(47.0 \pm 2.4 \mathrm{mas} / \mathrm{yr})$, almost exactly to the east, so that the separation to the companion candidate ( $3^{\prime \prime}$ north) would hardly change, even if it is a background object.

Before obtaining a spectrum, we took aquisition images, which can be used to measure the separation between primary star and companion candidate. This separation should be constant with time, if its a common proper motion pair; a change in separation due to orbital motion in case of a non-circular orbit would be negligible.

The companion candidate is detected marginally in the aquisition image on 10 July 2003, but detected clearly on 20 July 2003 (Fig. 1), obtained through a narrow band filter at $2.19 \mu \mathrm{m}$ in the $K$-band.

We compile the previous and new astrometric results in Table 1. There are images at three epochs available now, first detected on 04 July 2001 (N03), then on 29 Oct. 2001 (C03), and finally on 20 July 2003 (here). We measure the position of the primary star and the companion candidate in every co-added image with center/gauss in MIDAS, which provides

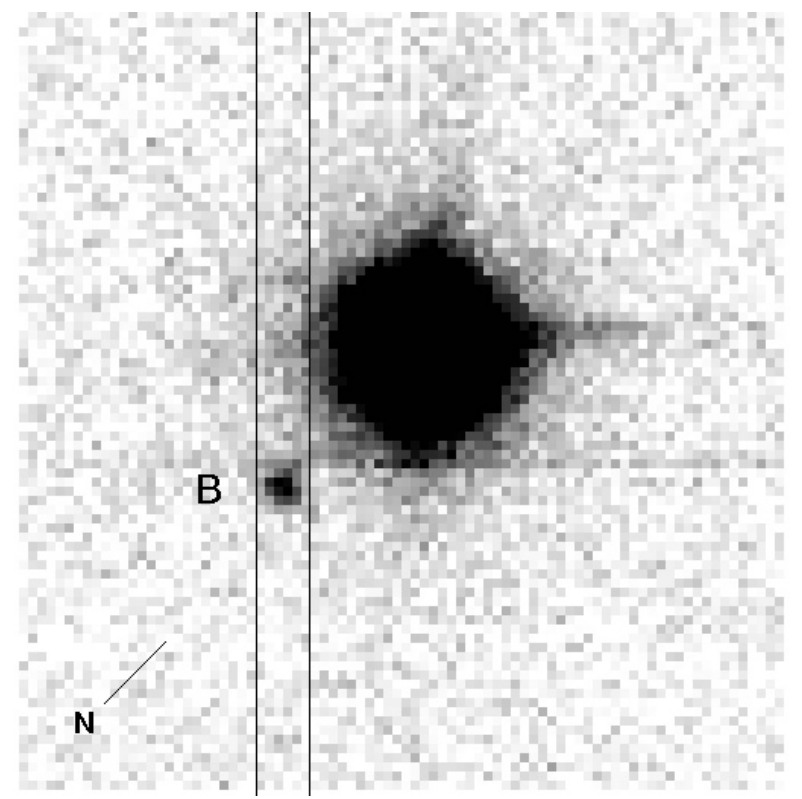

Fig. 1. Aquisition image through a narrow band filter in the $K$-band taken with ISAAC at the VLT before our spectroscopy, showing the companion candidate B three arcsec north of GSC 08047-00232 A. The $0.8^{\prime \prime}$ wide slit was located neither along both objects nor perpendicular to that direction, but inbetween the two, namely from SE (top) to NW (bottom) with the faint companion candidate in the slit and the bright primary star outside the slit, so that we still obtain some stray light from the bright star in the slit, which we use for calibration purposes. North is to the bottom left, the line is 1.5 arcsec long.

$1 \sigma$ errors. Pixel scales, detector orientation, and their errors are measured on calibration fields with many stars of known fixed positions taken in the same nights ${ }^{1}$. With these values, we can obtain separations and position angles (PA), see Table 1.

Our three measurements - separated by two years of the separation and the PA are consistent with eachother within $1.5 \sigma$. The companion candidate is located almost

\footnotetext{
1 While the measurement of the separation between the two object is very precise on the ISAAC aquisition image obtained in July 2003, we could not measure the position angle PA with high precision, see Table 1, because there are no other objects detected on the (short exposure) aquisition image, with whose positions we could have measured the actual detector orientation; hence, we have to rely on the header information, which we know to be less precise from previous experience; also, being just an aquisition image obtained in a service mode night, we do not have other images obtained at the same detector orientation of other fields, which could otherwise be used for such a calibration; hence, the large uncertainty in the third PA values in Table 1, while the separation is more precise being independant of the detector orientation.
} 
directly north of the primary star (only 1 to $2^{\circ}$ west of the north direction) and the primary star is known to move towards the east (just a bit south of east). Hence, the separation should increase with time, if the companion is a non-moving background star. Given the proper motion of the primary star, it should have increased to $3.25^{\prime \prime}$ by July 2003. As observed (Table 1), the separation has not changed within the errors. The astrometry is $2 \sigma$ deviant from a background hypothesis. However, due to the small proper motion and the small epoch difference, we cannot yet reliably distinguish between the companion candidate being either a common proper motion companion and a background star.

\section{Spectroscopic observations}

We obtained $H$ - and $K$-band spectra with the Infrared Spectrometer And Array Camera (ISAAC) at Antu, Unit Telescope 1 (UT1) of the Very Large Telescope (VLT) of the European Southern Observatory (ESO) on Cerro Paranal, Chile. ISAAC is equipped with a $1024 \times 1024$ HAWAII detector. All $H$ - and $K$-band spectra obtained have 60 s exposure each and are taken through a $0.8^{\prime \prime}$ slit with a low resolution of $R \simeq 1700$. In the $H$-band, centered on $1.65 \mu \mathrm{m}$, we took 28 spectra on 20 July 2003 . In the $K$-band, centered on $2.20 \mu \mathrm{m}$, we took 28 spectra on 11 July 2003 and again 28 spectra on 20 July 2003. All data were obtained in service mode. Calibration frames were taken in the same nights: darks, flats, and arcs for wavelength calibration.

We measured the full width at half maximum (FWHM) on the aquisition images as well as in the individual spectra obtaining $0.5^{\prime \prime}$ for both nights. The $S / N$ in the (unbinned) $K$-band spectrum taken on 20 July 2003 is $\sim 24$, but only $\sim 20$ in the data taken on 11 July 2003 , so that we use the $K$-band spectrum from 20 July 2003.

Data reduction was done in the normal way with IRAF: dark subtraction, normalization, flat fielding, sky subtraction, wavelength calibration, co-adding the spectra, then correction for instrumental sensitivity and atmospheric response. We use the spectrum of the bright primary star (located off the slit) for calibration purposes. The $S / N$ in the binned spectra is $\sim 40$ for both $H$ and $K$. For the new companion candidate as well as for the spectral templates HR 7329 and TWA 5 with their known companions, both the primary and the secondary are observed simultaneously, so that we can do almost perfect calibration of the continuum shapes by using the known spectral type of the primary for estimating the response of the detector and, hence, calibrating the secondary. This is different for the spectral template Cha $\mathrm{H} \alpha 10$. However, even with templates observed simultaneously, some uncertainties in the calibration remain, so that spectral types obtained from spectral indices should be more accurate than those obtained from continuum slopes.

The final co-added $H$ - and $K$-band spectra are shown in Figs. 2 to 4 respectively. We compare the spectra of GSC 08047-00232/cc with those of young late-M type BDs in Chamaeleon, Tucana, and TWA.

The $H$-band spectrum is compared with Cha $\mathrm{H} \alpha 10$ observed with ISAAC by us with the same set-up (in June 2000).

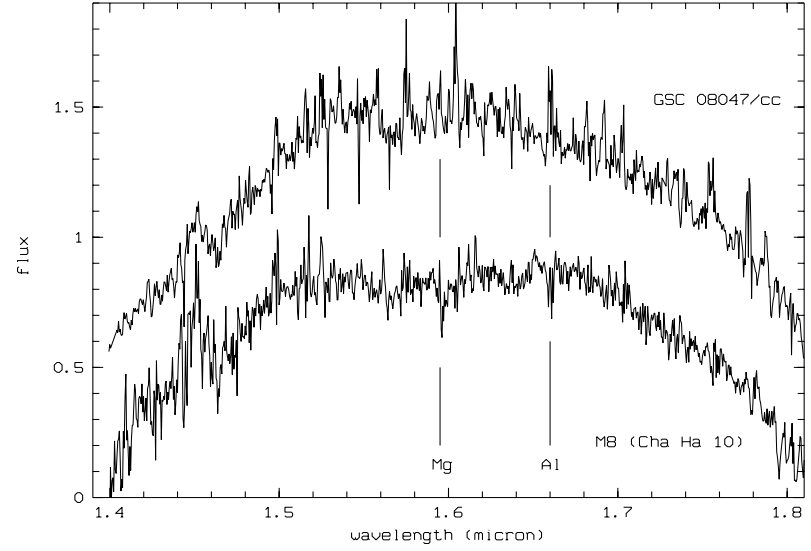

Fig. 2. $H$-band spectra of the companion candidate GSC 08047-00232/cc (top) compared to the H-band spectrum of the M 7.5-type young BDs Cha $\mathrm{H} \alpha 10$ (bottom) in Chamaeleon also observed by us with ISAAC with the same set-up (in June 2000). Both spectra are relatively featureless and show a similar continuum shape, so that the companion is similar to $\mathrm{Cha} \mathrm{H} \alpha 10$ in its spectral type ( \pm 1 sub-class, i.e. M $6.5-8.5$ ).

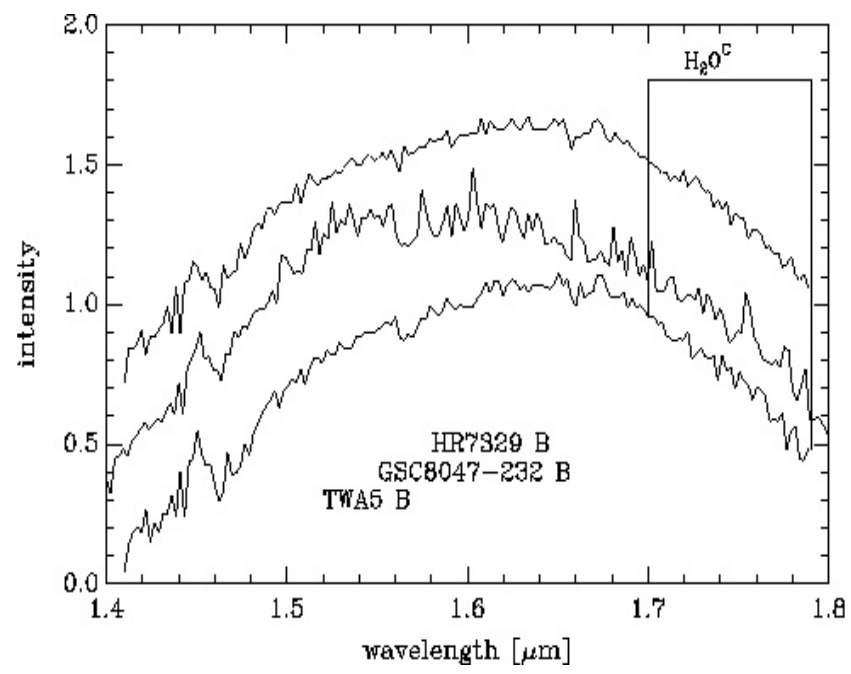

Fig. 3. $H$-band spectra of GSC 08047-00232/cc (middle) compared with HR 7329 B (M 7-8, top) and TWA-5 B (M 8.5-9, bottom). Here, we have smoothed the spectral resolution by a blocking factor of 5 yielding a resolution of $\sim 350$ and a $S / N$ ratio of $\sim 40$. The slope in the red part of the spectrum of GSC 08047-00232/cc is intermediate between that of HR 7329 B and TWA-5 B. The Reid et al. (2001) spectral index $\mathrm{H}_{2} \mathrm{O}-\mathrm{C}$ is most sensitive to the continuum slope and, hence, spectral type, indicated in the upper right, yielding roughly $\mathrm{M} 8$ as spectral type for GSC 08047-00232/cc.

Cha $\mathrm{H} \alpha 10$ is a young ( 2 Myr) M 7.5 BD in the Cha I star forming region (Comerón et al. 2000). Both spectra are relatively featureless with the same continuum shape indicative of a very similar spectral type. The slope in the red part of the $H$-band at $\sim 1.7$ to $1.8 \mu \mathrm{m}$ is very sensitive to the temperature at late $\mathrm{M}$ and $\mathrm{L}$ spectral types due to water absorption. As seen in Fig. 2, the red slope in GSC 08047-00232/cc is slightly steeper than in $\mathrm{Cha} \mathrm{H} \alpha$ 10; hence, GSC 08047-00232/cc appears slightly cooler, i.e. slightly later, around M 8-8.5, while the broad maximum of the flux in GSC 08047-00232/cc is at 


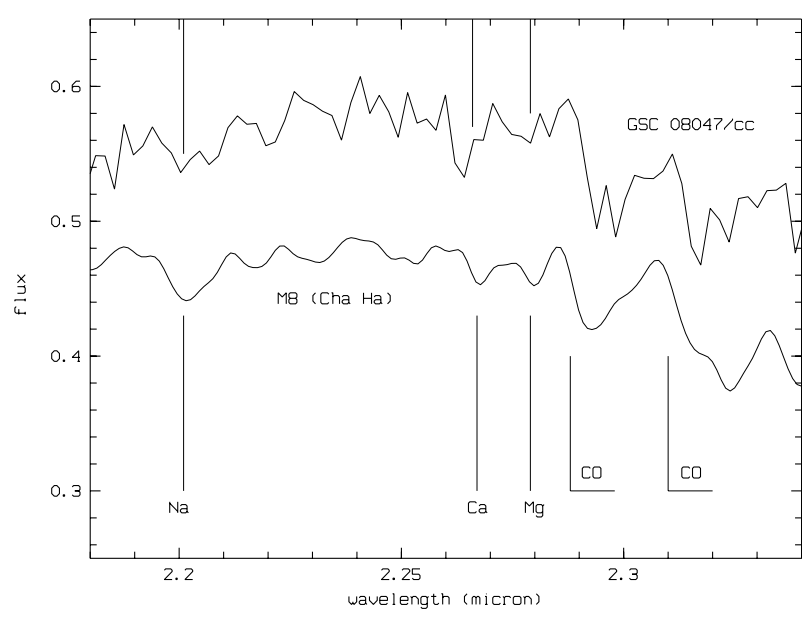

Fig. 4. Rebinned $K$-band spectra of the companion candidate GSC 08047-00232/cc (top) compared to the averaged $K$-band spectrum of M 7.5- to M 8-type young BDs (bottom) in Chamaeleon (from Comerón et al. 2000), obtained with SOFI, an IR spectrograph similar to ISAAC, but at a different spectral resolution. Both spectra show the same absorption lines $\mathrm{Na}, \mathrm{Ca}$, and $\mathrm{Mg}$, very similar molecular $\mathrm{CO}$ bands, and the same continuum shape, so that the companion is similar in spectral type as the template, i.e. M 7-8.5.

a slightly bluer wavelength compared to $\mathrm{Cha} \mathrm{H} \alpha 10$, which would indicate a slightly earlier spectral type, around M 6.5-7. Hence, we classify the companion candidate tentatively as M 6.5-8.5 (from the $H$-band continuum slopes).

Then, we estimate the spectral index $\mathrm{H}_{2} \mathrm{O}-\mathrm{B}$ following Reid et al. (2001), the ratio of the fluxes at 1.48 and $1.60 \mu \mathrm{m}$. We obtain $0.88 \pm 0.09$ for GSC 08047-00232/cc, hence M 8.5-9.5. Then, the spectral index $\mathrm{H}_{2} \mathrm{O}-\mathrm{C}$ is sensitive for the slope in the red part of the $H$-band from 1.7 to $1.8 \mu \mathrm{m}$, i.e. very sensitive to the spectral type; we obtain 0.56 for GSC 08047-00232/cc, 0.55 for TWA-5 B (M 8.5-9), and 0.58 for HR 7329 B (M 7-8). Hence, GSC 08047-00232/cc is intermediate between TWA-5 B and HR 7329 B and can be classified as M 8-8.5 (from $\mathrm{H}_{2} \mathrm{O}-\mathrm{C}$ ). Together with the above comparison of GSC 08047-00232/cc and the M 7.5-type Cha $\mathrm{H} \alpha 10$ yielding M 6.5-8.5 (Fig. 2), we classify GSC 08047-00232/cc as $\mathrm{M} 8 \pm 2$, from the $H$-band.

The $K$-band spectrum (Fig. 4) is compared with an averaged $K$-band spectrum of $\mathrm{M} 7.5$ - to $\mathrm{M}$ 8-type BDs in Chamaeleon (Comerón et al. 1999, 2000) obtained with SOFI, because we did not take a $K$-band spectrum of Cha $\mathrm{H} \alpha 10$ or any other M 6-8 type Chamaeleon BD with ISAAC. Here, we see again a very similar continuum shape as well as several lines $(\mathrm{Na}, \mathrm{Ca}$, and $\mathrm{Mg}$ ) similar in both spectra. Also, the $\mathrm{CO}$ molecular absorption bands are very similar in both spectra, so that we can classify GSC $08047-00232 / \mathrm{cc}$ as roughly M 8 from the $K$-band, consistent with the $H$-band. The $K$-band spectrum of GSC $08047-00232 / \mathrm{cc}$ is clearly later than the average M 6 and average M 8-type spectra from Comerón et al. (1999).
To summarize, the companion candidate can be classified as M $8 \pm 2$ (or M 6-M 9.5) from the $H$ - and $K$-band spectra. This classification is tentative and rough, because of the relatively low spectral resolution, low $S / N$ ratio.

\section{Result: A brown dwarf companion}

Obviously, it is very unlikely to find by chance an object with spectral type M $8 \pm 2$ just 3 " off one of our targets. According to the Besancon galactic model, to find an object as faint as GSC $08047-00232 / \mathrm{cc}$ within $3^{\prime \prime}$ of the primary star at the given galactic latitude is only $\sim 0.4 \%$ (C03) with sample sizes being 25 stars in N03 and 23 stars in C03 with 13 overlaps; it is even less likely, to find such an object with (at least roughly) the same proper motion (see Table 1) and the late M spectral type. Hence, the companion candidate most certainly is a truely bound companion. For the remainder of the paper, we regard this companion candidate as a bound companion of the star and call it GSC 08047-00232 B.

The primary star GSC 08047-00232 A, as a probable member of $\sim 35 \mathrm{Myr}$ young TucHorA, has a distance of $\sim 50 \mathrm{pc}$ (Torres et al. 2000; Zuckerman \& Webb 2000) to as much as $\sim 85 \mathrm{pc}(\mathrm{C} 03)$. The larger distance is obtained, when forcing the star to be co-eval with the rest of the TucHorA stars at $\sim 35 \mathrm{Myr}$ with the Baraffe et al. (1998) models using the hydrostatic scale-height $1.0 H_{\mathrm{p}}$ as mixing length parameter. However, with e.g. $1.9 H_{\mathrm{p}}$, the star is again older. On D'Antona \& Mazzitelli (1998) tracks, the star is 30 to 50 Myr young at 85 pc, see Fig. 5. Hence, we assume $85 \mathrm{pc}$ as distance and $\sim 35 \mathrm{Myr}$ as age for both the primary star and its companion.

Given the spectral type M $8 \pm 2$ (Sect. 3), we obtain $\sim 2100 \mathrm{~K}$ as temperature following Jones \& Tsuji (1997) and Kirkpatrick et al. (1999), or $\sim 2500 \mathrm{~K}$ from the Luhman (1999) extrapolation of the Leggett et al. (1996) scale for dwarfs ${ }^{2}$, so that we use $2300 \pm 250 \mathrm{~K}$ as temperature.

The companion has an absolute magnitude of $M_{K}=10.2 \pm 0.1$ at $85 \mathrm{pc}$. According to $J-K=$ $1.35 \pm 0.32 \mathrm{mag}(\mathrm{C} 03)$ and the spectral type M 6-9.5, we use a bolometric correction in the $K$-band of $B C_{K}=3.0 \pm 0.1 \mathrm{mag}$ following Leggett et al. (2002). With $K=14.9 \pm 0.1 \mathrm{mag}$ (C03, N03), we then obtain a bolometric luminosity of $\log \left(L_{\mathrm{bol}} / L_{\odot}\right)=-3.3 \pm 0.1$ for $85 \mathrm{pc}$ (or $-3.85 \pm 0.10$ for $50 \mathrm{pc}$ ). With these values for temperature, luminosity, and age, we can obtain a mass estimate using theoretical model calculations, see Table 2.

Using Fig. 7 in Burrows et al. (1997) for $\log \left(L_{\mathrm{bol}} / L_{\odot}\right)=-3.3$ at $35 \mathrm{Myr}$, we obtain $\sim 20$ to $30 M_{\text {jup }}$, from Fig. 9 for $2300 \pm 250 \mathrm{~K}$ at $35 \mathrm{Myr}$, we derive $\sim 15$ to $35 M_{\text {jup }}$, and from Fig. 11 for $\log \left(L_{\mathrm{bol}} / L_{\odot}\right)=-3.3$ and $2300 \pm 250 \mathrm{~K}$, we get $\sim 20$ to $40 M_{\text {jup }}$. Using Fig. $2(35 \mathrm{Myr}$ ) in Chabrier et al. (2000) with $T=2300 \pm 250 \mathrm{~K}$ at $35 \mathrm{Myr}$, we obtain $\sim 20$ to $40 M_{\text {jup }}$; from Table 1 in Chabrier et al. (2000) (i.e. for $100 \mathrm{Myr}$ as their youngest age) and using $T=2300 \pm 250 \mathrm{~K}$,

\footnotetext{
${ }^{2}$ Because the object is close to the main sequence, it is much closer to true dwarfs than to objects intermediate between dwarfs and giants, so that we use the dwarf scale instead of Luhman's intermediate scale; for intermediate objects, the temperature would be $\sim 2635 \mathrm{~K}$.
} 


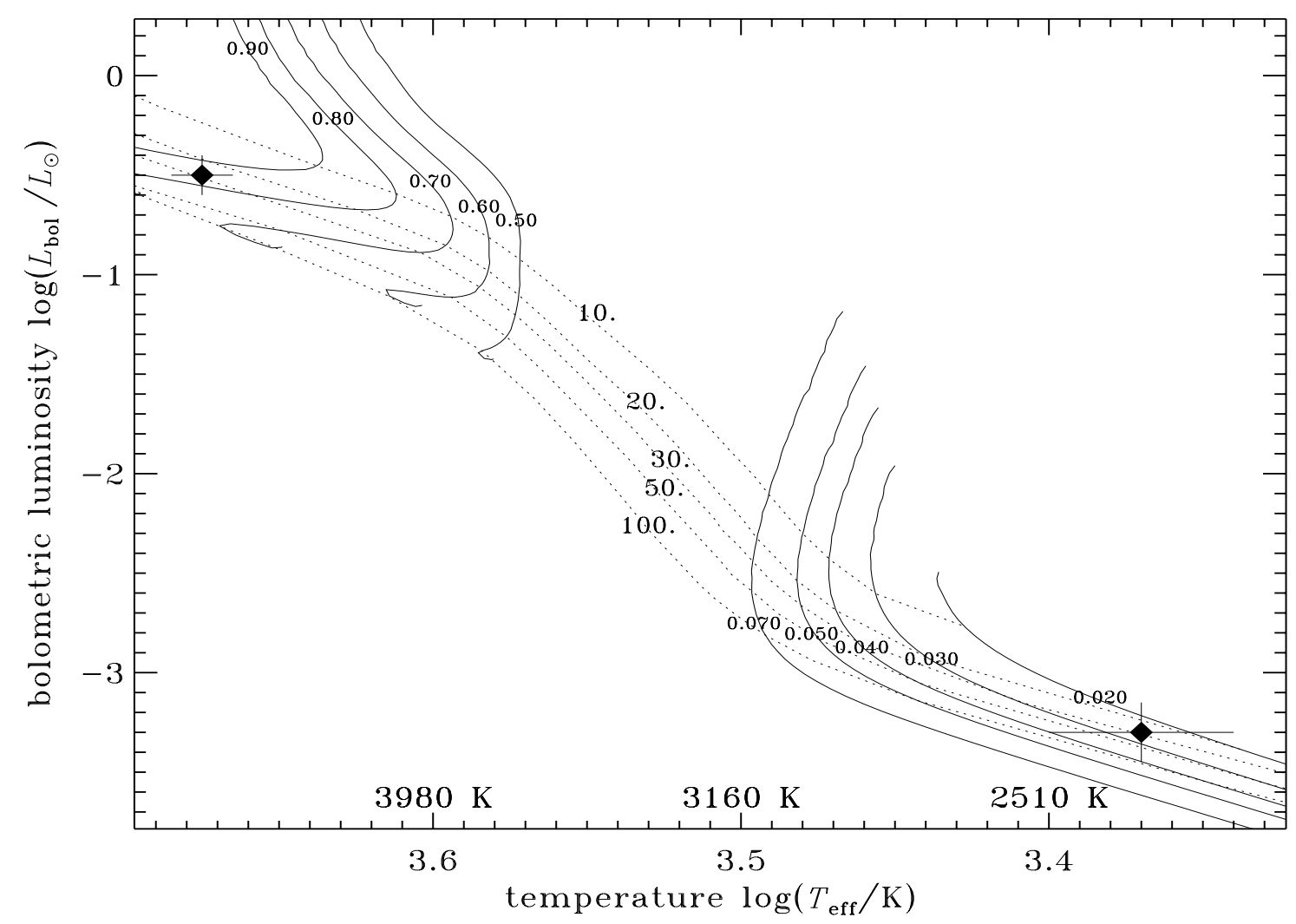

Fig. 5. H-R diagram for GSC 08047-0232 A and B (at 85 pc) with theoretical tracks and isochrones from D'Antona \& Mazzitelli (1998), ages in Myr, masses in solar masses. The objects are co-eval at 30 to $50 \mathrm{Myr}$.

Table 2. Mass determination for the companion ( $M_{\mathrm{j}}$ for Jupiter mass).

\begin{tabular}{lll}
\hline \hline Model & Parameters used & Mass \\
\hline Burrows et al. (1997), Fig. 7 & $35 \mathrm{Myr}, L$ at $85 \mathrm{pc}$ & $20-30 M_{\mathrm{j}}$ \\
Burrows et al. (1997), Fig. 9 & $T=2300 \mathrm{~K}, 35 \mathrm{Myr}$ & $15-35 M_{\mathrm{j}}$ \\
Burrows et al. (1997), Fig. 11 & $T=2300 \mathrm{~K}, L$ at $85 \mathrm{pc}$ & $20-40 M_{\mathrm{j}}$ \\
Chabrier et al. (2000), Fig. 2 & $T=2300 \mathrm{~K}, 35 \mathrm{Myr}$ & $20-40 M_{\mathrm{j}}$ \\
Chabrier et al. (2000), Table 1 & $T, L$ and $M_{K}$ at $85 \mathrm{pc}$ & $35-45 M_{\mathrm{j}}$ \\
Baraffe et al. (2002), Fig. 2 & $T, 35 \mathrm{Myr}, L$ at $85 \mathrm{pc}$ & $\sim 20 M_{\mathrm{j}}$ \\
Baraffe/Chabrier in C03 & $M_{K}$ vs. $J-K$ CMD & $20-40 M_{\mathrm{j}}$ \\
Wuchterl \& Tscharnuter (2003) & $T, L$ at $85 \mathrm{pc}, 35 \mathrm{Myr}$ & $\sim 7 M_{\mathrm{j}}$ \\
D'Antona \& Mazzitelli (1998) & $T, L$ at $85 \mathrm{pc}($ Fig. 5) & $20-50 M_{\mathrm{j}}$ \\
\hline
\end{tabular}

$\log \left(L_{\mathrm{bol}} / L_{\odot}\right)=-3.3$, and $M_{K}=10.2 \pm 0.1 \mathrm{mag}$, we obtain $\sim 35$

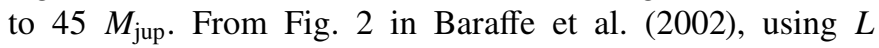
and $T$ as above at $35 \mathrm{Myr}$, we obtain a mass of $\sim 20 M_{\text {jup }}$. On D’Antona \& Mazzitelli (1998) tracks and isochrones, the primary star and the companion are co-eval at $85 \mathrm{pc}$ with an age of $\sim 20$ to $50 \mathrm{Myr}$ and with a mass of $\sim 20$ to $50 M_{\text {jup }}$ for the companion (see Fig. 5).

C03 show a color-magnitude diagram (CMD), $M_{K}$ versus $J-K$ (their Fig. 4), for the primary star with its companion candidate; they compare their locations in the CMD at 60 and $85 \mathrm{pc}$ distances with model calculations made by Baraffe et al. (1998) and Chabrier et al. (2000) for 20 to $50 \mathrm{Myr}$; they find the two objects to be co-eval for 85 pc only (at 40 Myr) with the mass of the companion then being $\sim 20$ to $40 M_{\text {jup }}$. Hence, with both the D'Antona \& Mazzitelli (1998) model and the Baraffe et al. (1998) model, we consistently obtain $35 \mathrm{Myr}$ and $\sim 30 M_{\text {jup }}$ for the companion.

We have to caution, however, as discussed in Wuchterl (2001) and Baraffe et al. (2002), that theoretical models are more uncertain for young ages (even tens of Myr) as compared to older (Gyr) ages, because of uncertain initial conditions. Hence, there could easily be a systematic shift in our mass estimates. Extrapolating the Wuchterl \& Tscharnuter (2003) models by scaling the luminosity dependance in mass and age (to $35 \mathrm{Myr}$ ), one would obtain a mass estimate of only $\sim 7 M_{\text {jup }}$, below the deuterium burning mass limit (G. Wuchterl, private communication), which we will study and discuss in more detail elsewhere. 
To summarize, we obtain a mass estimate of $\sim 15$ to $50 M_{\text {jup }}$ with $\sim 25 M_{\text {jup }}$ as most likely value, from conventional models by the D'Antona \& Mazzitelli, Chabrier \& Baraffe, as well as Burrows et al. groups, but possibly a lower mass when formation scenarios are taken into account. GSC 08047-00232 B is probably a low-mass brown dwarf. To confirm companionship of this late-M type object with the primary star beyond any doubt, common proper (or radial) motion (or reflex motion of the primary due to the companion) or orbital motion ( $\sim 3000$ year orbit with $\sim 250$ AU semi-major axis) would have to be shown with high significance.

If the primary star GSC 08047-00232 would not be a member of HorA or TucHorA, the assumed distance for primary and companion could be wrong. However, the strong lithium absorption line (together with the other youth indicators like strong X-ray emission, $\mathrm{H} \alpha$ emission, and fast rotation) clearly indicate that the primary star is a pre-main sequence star, so that the spectral type of the companion, $\mathrm{M} 8 \pm 2$, alone points to a mass near or below the $\mathrm{H}$ burning limit.

\section{The frequency of brown dwarf companions}

Most young stars in the nearby TWA, TucHorA, and $\beta$ Pic associations have been surveyed by deep IR imaging (with speckle and/or AO and/or HST) for substellar companions, so that we can try to do some statistics:

in TWA, there are 19 member systems (see Torres et al. 2003 for a recent list) including visual multiples, so that there is a total number of 27 stars. All of them have been surveyed by now with one BD companion found and confirmed, namely TWA-5 B (Lowrance et al. 1999; Neuhäuser et al. 2000). The sensitivity of the surveys are deep enough to have detected all $\mathrm{BD}$ companions down to the $\mathrm{D}$ burning limit outside of roughly $50 \mathrm{AU}$; however, they are quite inhomogenous, done by several groups with several different telescopes and instruments. There is a total of one BD companion among 27 stars, which is a frequency of $4 \pm 4 \%$.

With imaging, one can detect only those companions separated from the primary in the two dimensions of the plane of the sky, i.e. those separated well in right ascension $\alpha$ and/or declination $\delta$. One cannot detect those companions directly (or almost directly) in front of or behind the primary, i.e. located (almost) on the same line-of-sight as the primary, instead of having an angular separation. By direct imaging, one can detect companions only in the two dimensions $\alpha$ and $\delta$, not in the 3 rd dimension ${ }^{3}$. To correct the number above (from two to three dimenions, $2 \mathrm{D}$ to $3 \mathrm{D}$ ), we have to add $\sim 1 / 2$ of the $2 \mathrm{D}$ value; hence, we obtain $\sim 6 \pm 6 \%$ (for TWA).

In the $\beta$ Pic association, Neuhäuser et al. (2000) have done a homogenous survey for companions down to the D burning limit outside of $~ 50$ AU. Before, one BD companion was known, namely HR 7329 B (Lowrance et al. 2000; Guenther et al. 2001). Neuhäuser et al. (2000) did not find any additional BD companion (or candidates) among a total of 17 stars

\footnotetext{
3 In radial velocity searches, one can detect all companions, unless the orbital plane is perpendicular to the line-of-sight.
}

in 12 systems. Hence, the frequency of BD companions in $\beta$ Pic is $6 \pm 6 \%$ (or $9 \pm 9 \%$ after correcting from $2 \mathrm{D}$ to $3 \mathrm{D}$ ).

In TucHorA, 25 stars were observed by Neuhäuser et al. (2000) and ten more by Chauvin et al. (2003), both down to roughly the D burning limit for companions outside of 50 AU. The number of $\mathrm{BD}$ companions here is again one, namely the one confirmed above. Hence, here we have a frequency of BD companions of $3 \pm 3 \%$ (or $4 \pm 4 \%$ for $3 \mathrm{D}$ ).

In all three associations, the frequency of BD companions (of any mass down to the $\mathrm{D}$ burning limit of $\sim 13 M_{\text {jup }}$ ) outside of roughly $50 \mathrm{AU}$ separation is very low, around a few per cent. The spectral types of the primaries ranges from A0 (HR 7329 A) over K3 (here, GSC 08047-00232 A) to M2 (TWA-5 A). Its still low number statistics with one BD companion each, but since 17 to 35 stars have been observed, one can clearly say that the frequency of wide BD companions ( $\geq 50 \mathrm{AU}$ ) is quite low. Putting together the three associations, we have three BDs around 79 stars, i.e. a frequency of $4 \pm 3 \%$ (or $6 \pm 4 \%$ after correction of the projection effect from $2 \mathrm{D}$ to $3 \mathrm{D}$ ), which is the best estimate so far for the frequency of BDs (of any mass) outside of $50 \mathrm{AU}$ around $\sim 10$ to 35 Myr young stars born in loose associations. This value is consistent with the expectation, if the mass function of binaries is similar to the mass function of field stars, in which case $\sim 3$ to $12 \%$ of stars should have BD companions (see Gizis et al. 2001), so that there seems to be no evidence for a BD desert at wide separation.

Acknowledgements. We would like to thank the ESO Service Mode observing team on Cerro Paranal as well as the ESO User Support Group for perfect support in both observing periods 65 and 71. M. Ammler and T. Schöning prepared Fig. 5. We also acknowledge Wolfgang Brandner and Günther Wuchterl for many stimulating discussions.

\section{References}

Baraffe, I., Chabrier, G., Allard, F., \& Hauschildt, P. H. 2002, A\&A, 382,563

Bouvier, J., Duchene, G., Mermilliod, J. C., \& Simon, T. 2001, A\&A, 375, 989

Burrows, A., Marley, M., Hubbard, W. B., et al. 1997, ApJ, 491, 856

Chabrier, G., Baraffe, I., Allard, F., \& Hauschildt, P. H. 2000, ApJ, 542,464

Chauvin, G., Thomson, M., Dumas, C., et al. 2003, A\&A, 404, 157 (C03)

Comerón, F., Rieke, G. H., \& Neuhäuser, R. 1999, A\&A, 343, 477

Comerón, F., Neuhäuser, R., \& Kaas, A. A. 2000, A\&A, 359, 269

D'Antona, F., \& Mazzitelli, I. 1998, A Role for Superadiabatic Convection in Low Mass Structures? in Brown dwarfs and extrasolar planets, ed. R. Rebolo, E. L. Martin, \& M. R. Zapatero-Osorio, Proc. Workshop held in Puerto de la Cruz, Tenerife, Spain, 17-21 March 1997, ASP Conf. Ser., 134, 442

Geballe, T. R., Knapp, G. R., Leggett, S. K., et al. 2002, ApJ, 564, 466

Gizis, J. E., Kirkpatrick, J. D., Burgasser, A., et al. 2001, ApJ, 551, L163

Guenther, E. W., Neuhäuser, R., Huélamo, N., Brandner, W., \& Alves, J. 2001, A\&A, 365, 514

Hog, E., Fabricius, C., Makarov, V. V., et al. 2000, A\&A, 355, L27

Jones, H. R. A., \& Tsuji, T. 1997, ApJ, 480, L39

Kenyon, S. J., \& Hartmann, L. W. 1995, ApJS, 101, 117 
Kirkpatrick, J. D., Reid, I. N., Liebert, J., et al. 1999, ApJ, 519, 802 Leggett, S. K., Allard, F., Berriman, G., Dahn, C. C., \& Hauschildt, P. H. 1996, ApJS, 104, 117

Leggett, S. K., Golimowski, D. A., Fan, X., et al. 2002, ApJ, 564, 452

Lowrance, P. J., McCarthy, C., \& Becklin, E. E. 1999, ApJ, 512, L69

Lowrance, P. J., Schneider, G., Kirkpatrick, J. D., et al. 2000, ApJ, 541, L390

Luhman, K. 1999, ApJ, 525, 466

Neuhäuser, R., Guenther, E. W., Brandner, W., et al. 2000, A\&A, 360, L39

Neuhäuser, R., Guenther, E. W., Alves, J., et al. 2003, AN, 324, 535 (N03)

Patience, J., Ghez, A. M., Reid, I. N., \& Matthews, K. 2002, AJ, 123, 1570
Reid, I. N., Burgasser, A. J., Cruz, K. L., Kirkpatrick, J. D., \& Gizis, J. E. 2001, AJ, 121, 1720

Reipurth, B., \& Clarke, C. 2001, AJ, 122, 432

Torres, C. A. O., da Silva, L., Quast, G. R., de la Reza, R., \& Jilinski, E. 2000, AJ, 120, 1410

Torres, G., Guenther, E. W., \& Marschall, L. A. 2003, AJ, 125, 825

Voges, W., Aschenbach, B., Boller, Th., et al. 1999, A\&A, 349, 389

Webb, R. A., Zuckerman, B., Platais, I., et al. 1999, ApJ, 512, L63

Wuchterl, G. 2001, A Dialogue on Dynamical Pre-Main Sequence Tracks, in The Formation of binary stars, IAU Symp., 200, 492

Wuchterl, G., \& Tscharnuter, W. M. 2003, A\&A, 398, 1081

Zuckerman, B., \& Webb, R. A. 2000, ApJ, 535, 959 